\title{
Performance Evaluation by Measurement Combination of Loosely Coupled GPS/INS Integration
}

\author{
Daero Lee ${ }^{1,2}$ \\ 1 Research Center for Aircraft Core Technology, Gyeongsang National University, Jinju, Republic of \\ Korea; leed61@naver.com \\ 2 Aerospace Science Engineering, Tuskegee University, Tuskegee, AL, USA; dlee@ tuskegee.edu \\ * Correspondence: leed61 @ naver.com; Tel.: +82-10-5052-3885
}

\begin{abstract}
This paper presents an extended Kalman filter derivation for loosely coupled GPS (Global Positioning System)/INS (Inertial Navigation System) integration based on quaternion attitude representation using the Earth-Centered Earth (ECEF) Frame. In this loosely coupling integration, both the position and velocity estimates from GPS receiver are used as the measurements to extended Kalman filter, and then they are integrated with inertial measurements from inertial measurement units (IMU) to estimate the attitude, position and velocity of an air vehicle. The velocity estimates which have centimeter level estimation error from the GPS receiver are used to improve the filter performance. For attitude estimation, the global attitude parameterization is given by a quaternion and a multiplicative quaternion-error approach is used to guarantee a normalization constraint of quaternion in the filter. Simulation results are shown to obtain the estimation of the attitude, position, velocity, biases and scale factors and to evaluate the performance of the EKF with the measurement combination composed of the two different types of GPS navigation solutions (the position only, and both the position and the velocity in the ECEF frame).
\end{abstract}

Keywords: loosely coupled GPS/INS integration; Earth-Centered Earth-Fixed Frame; multiplicative quaternion-error approach; GPS navigation solutions; extended Kalman filter

\section{Introduction}

Integration of GPS (Global Navigation Satellite System) and INS (Inertial Navigation System) has been intensively studied and widely applied, and become a standard method for position and attitude estimation of a moving vehicle due to their complementary characteristics [1,2,3,4]. GPS and INS (GPS/INS) integration aims to utilize the advantages of the two individual systems and make up for their weaknesses. An INS is a self-contained system regardless of the operational environment and provides high frequency information by mechanizing the acceleration and angular rate outputs of the IMU (Inertial Measurement Unit) which consists of three orthogonal accelerometers and three orthogonal gyros [5]. MEMS (Microelectromechanical systems) technology can reduce the IMU's cost considerably, it could make IMU's results diverge after continuously using for a long period of time. On the other hand, GPS is an external navigation system that can provide low frequency navigation information (acceleration and rotation rate of the object) with bounded error over time [5]. By aiding the INS with external measurements related to one or several of the system states, INS errors can be estimated and corrected for, e.g. by using a Kalman filter. The integration of GPS with INS improves the quality and integrity of each navigation system: use of GPS permits calibration of inertial instrument biases, and the INS can be used to improve the tracking and reacquisition performance of the GPS receiver. The benefits of a GPS/INS integration is that the INS estimates can be corrected by the GPS data and that the INS can provide 
position and angle updates at a higher rate than GPS. The aiding measurements can be processed in a separate filter and included in the INS filter in the form of position and velocity measurements, so-called "loosely coupled" integration. Alternatively, aiding measurements, e.g. GPS pseudorange, may be included directly into the INS filter, so-called "tightly coupled" integration. The loosely coupled GPS/INS integration strategy is common to use due to its faster processing, simplicity of implementation and robustness. In loosely coupled integration, the GPS position and velocity solutions are used as measurement inputs to the integration filter which estimates the INS errors [4,5]. The navigation solution of the loosely coupled integration is the INS navigation solution, corrected with the Kalman filter. While four or more satellites are visible to GPS receiver, GPS position/velocity solution can be obtained and loosely coupled integration can offer high system robustness. The principle advantage of loosely coupled integration is simplicity with the chosen computational frame. Loosely coupled integration can operate with GPS position measurements alone or velocity measurements alone [6,7].

The GPS velocity measurement obtained by differentiating GPS position measurement is noisy when a code-based sing-epoch GPS position is used [4,8]. On the other hand, the GPS velocity measurement of a carrier-smooth-code based or Kalman filter-based solution is less noisy than the above one. One notable aspect of GPS/INS integration is the coordinate system used to describe the determined position and velocity. An Earth-Centered Earth-Fixed (ECEF) frame is a commonly used reference frame and resolving axes for the computation of satellite navigation solutions. The ECEF frame is usefully applied for a loosely coupled GPS/INS integration filter without any coordinate transformation since GPS receivers generally estimates the position and velocity in the same frame directly. In the navigation filter the ECEF frame has no need to take the covariance mappings from the NED (North-East-Down) to the ECEF frame and vice versa due to the difference of the coordinates while the NED frame requires covariance mappings [3]. However, the ECEF frame has a disadvantage in understanding the attitude of an air or ground vehicle intuitively while the NED frame provides more intuitive in expressing them as a local frame. The choice of the computation frame is not necessarily the most important factor in terms of computational efficiency although the navigation solutions are required in the geographical frame.

Various studies have been investigated for loosely coupled GPS/INS integration to address position and attitude estimation problem where most of them have been studied in the NED frame. Falco et. Al [9] investigated approaches for loosely coupled GPS/INS integration in the NED frame. Wei and Schwarz [10] developed two strapdown inertial algorithms using the local-level frame and ECEF frame, and compared them in terms of computation time. The algorithm using the ECEF frame is known to have about 30 percent more efficient than the standard algorithm using the local frame as long as an output rate of $5 \mathrm{~Hz}$ is sufficient [9]. Crassidis [3,4] showed an EKF (Extended Kalman Filter) and sigma-point Kalman filtering for integrated GPS (Global Positioning System) and inertial navigation in the NED frame using only GPS position measurement. The EKF is based on a quaternion parameterization of the attitude [3]. Edwan et al. [11] derived a total state loosely coupled GPS/INS integration algorithm based on the DCM (direction cosine matrix) attitude representation as a state space vector to be estimated. Gross and et al. [12] presented the EKF and UKF (Unscented Kalman Filter) implementations of GPS/INS integration algorithms in the geodetic frame for Euler angles based attitude, position and velocity estimations with the GPS position and velocity measurements, and evaluated with respect to performance and complexity. To represent attitude, several parameterizations can be used to represent such as Euler angles, quaternions, modified Rodrigues parameters, and the rotation vector. Quaternions are particularly appealing since no singularities are present and the kinematics equation is linear [13]. 
In this paper, an EKF filter using the Earth-Centered Earth-Fixed (ECEF) frame formulation is derived for loosely coupled GPS/INS integration to determine both the position and the attitude of an unmanned aerial vehicle (UAV). The GPS navigation solutions (position and velocity in the ECEF frame) are directly integrated with inertial measurements from gyros and accelerometers without coordinate transformation for loosely coupled GPSIINSS integration in the ECEF frame. This paper was motivated by the advantage of the ECEF frame for loosely coupled GPS/INS integration so that GPS navigation solutions are directly used as the EKF filter measurements in the ECEF-frame navigation equations without coordinate transformation. Furthermore, the global attitude parameterization expressed by a quaternion is used while a generalized three-dimensional attitude representation is used to define the local attitude error. A loosely coupled GPS/INS integration algorithm is easy to implement and needs to be derived with the following characteristics [7]. 1) Firstly, loosely coupled GPS/INS integration algorithm should be described using the ECEF frame so that the EKF can estimate the position, velocity and attitude of an UAV with respect to the ECEF frame using the GPS navigation solutions. 2) Secondly, centimeter per second-level of accuracy GPS velocity solutions are assumed to be available to the EKF as measurements as well as standalone GPS position solutions since recently a commercial GPS receiver can provide very precise velocity estimates [14]. 3) Thirdly, the EKF uses a multiplicative error quaternion after neglecting higher-order terms for attitude estimation since the attitude kinematics here is based on the quaternion, which must obey a normalization constraint that can be violated by the linear measurement updates associated with the standard EKF [4,13]. Then the four-component quaternion can effectively be replaced by a three-component error vector [15]. As a simulation result, the three different types of the GPS navigation solutions composed of the position solution only, and both the position and velocity solutions are used for the loosely coupled GPS/INS integration algorithm to analyze the estimation performances.

Thus, the main contribution of this paper is the derivation of the EKF for the loosely coupled GPS/INS integration algorithm so that the benefits of the ECEF frame, highly precise GPS velocity estimates and the quaternion parameterization can be used simultaneously with the measurement combination. Since the GPS navigation solutions are given in the ECEF frame, they are directly used to update the INS-derived trajectory with no coordinate transformation in the filter. In addition, the derived EKF based on the ECEF frame navigation equations has the benefit of faster computation compared to the filter based on the local navigation frame equations [10]. The derived EKF can use two different kinds of GPS navigation solutions composed of the position, and both the position and the velocity as the measurements obtained from the GPS receiver to the EKF. The loosely coupled GPS/INS integration algorithm can attain improvement in the filter performance when centimeter per second-level of accuracy GPS velocity solutions are available under the normal visibility of the GPS.

\section{Attitude Kinematics}

In this section the basic properties of attitude kinematics is summarized. One of the most useful attitude parameterizations is given by the quaternion, which is a four-dimensional vector. Like the Euler axis/angle parameterization, the quaternion is also four-dimensional vector, defined as $\mathbf{q} \equiv\left[\begin{array}{ll}\boldsymbol{\rho}^{T} & q_{4}\end{array}\right]^{T}$, with $\boldsymbol{\rho}=\left[\begin{array}{lll}q_{1} & q_{2} & q_{3}\end{array}\right]^{T}=\hat{\mathbf{e}} \sin (\vartheta / 2), \quad q_{4}=\cos (\vartheta / 2)$ where $\hat{\mathbf{e}}$ is the axis of rotation and $\vartheta$ is the angle of rotation [15]. The quaternion components are dependent each other since a four-dimensional vector is used to describe three dimensions. The quaternion satisfies a single constraint given by $\mathbf{q}^{T} \mathbf{q}=1$. To describe the attitude in different reference frames, superscripts and subscripts $i, e, n$ and $b$ which denotes the 
Earth-Centered-Inertial (ECI), ECEF, navigation and body frames are used in the attitude matrices. The attitude matrix that transforms the ECEF frame, $E$, to the body frame, $B$, is related to the quaternion by

$$
\begin{gathered}
C_{e}^{b}(\mathbf{q})=\Xi^{T}(\mathbf{q}) \Psi(\mathbf{q}) \\
\text { with } \Xi(\mathbf{q})=\left[\begin{array}{c}
q_{4} I_{3 \times 3}+[\boldsymbol{\rho} \times] \\
-\boldsymbol{\rho}^{T}
\end{array}\right], \quad \Psi(\mathbf{q})=\left[\begin{array}{c}
q_{4} I_{3 \times 3}-[\boldsymbol{\rho} \times] \\
-\boldsymbol{\rho}^{T}
\end{array}\right]
\end{gathered}
$$

where $I_{3 \times 3}$ is a $3 \times 3$ identity matrix and $[\times]$ is a notation for a cross product matrix [11].

For small angles the vector part of the quaternion is equal to half angles so that $\boldsymbol{\rho} \approx \boldsymbol{\alpha} / 2$ and $q_{4}$, where $\boldsymbol{\alpha}$ is a vector of the roll, pitch and yaw angles. The attitude matrix can then be approximated by $C_{e}^{b} \approx I_{3 \times 3}-[\boldsymbol{\alpha} \times]$ which is valid to within first-order in the angles.

The attitude kinematics equation is given by

$$
\dot{C}_{e}^{b}=-\left[\boldsymbol{\omega}_{e b}^{b} \times\right] C_{e}^{b}
$$

with

$$
\boldsymbol{\omega}_{e b}^{b}=\boldsymbol{\omega}_{i b}^{b}-C_{e}^{b}(\mathbf{q}) \boldsymbol{\omega}_{i e}^{e}
$$

where $\boldsymbol{\omega}_{e b}^{b}$ is the angular velocity of the $B$ frame relative to the $E$ frame expressed in $B$ coordinates, $\boldsymbol{\omega}_{i b}^{b}$ is the angular velocity of the $B$ frame relative to the $I$ frame expressed in $B$ coordinates and $\boldsymbol{\omega}_{i e}^{e}$ is the angular velocity of the Earth relative to the ECI frame expressed in the ECEF frame. Another form of Eq. (3) is given by

$$
\dot{C}_{b}^{e}=C_{b}^{e}\left[\boldsymbol{\omega}_{e b}^{b} \times\right]
$$

where $C_{b}^{e}=\left(C_{e}^{b}\right)^{T}$. The quaternion kinematics equation is given by

$$
\begin{array}{r}
\dot{\mathbf{q}}=\frac{1}{2} \Xi(\mathbf{q}) \boldsymbol{\omega}_{e b}^{e}=\frac{1}{2} \Omega_{e b}^{b}\left(\boldsymbol{\omega}_{e b}^{e}\right) \mathbf{q} \\
\text { with } \quad \Omega_{e b}^{b}\left(\boldsymbol{\omega}_{e b}^{e}\right)=\left[\begin{array}{cc}
-\left[\boldsymbol{\omega}_{e b}^{e} \times\right] & \boldsymbol{\omega}_{e b}^{e} \\
-\left(\boldsymbol{\omega}_{e b}^{e}\right)^{T} & 0
\end{array}\right]
\end{array}
$$

where $\boldsymbol{\omega}_{e b}^{e}$ is the angular velocity of the $B$ frame relative to the $E$ frame expressed in $B$ coordinates. A major benefit of using quaternions is that the kinematics equation is linear in the quaternion and is also free of singularities. Another advantage of quaternion is that successive rotations can be achieved using quaternion multiplication. Here the convention of Lefferts et al. [16] who multiply the quaternions in the same order as the attitude matrix multiplication (in contrast to the general convention established by Hamilton [16]) is adopted. This is written by

$$
C\left(\mathbf{q}^{\prime}\right) C(\mathbf{q})=C\left(\mathbf{q}^{\prime} \otimes \mathbf{q}\right)
$$

The composition of the quaternion is bilinear, with

$$
\mathbf{q}^{\prime} \otimes \mathbf{q}=\left[\Psi\left(\mathbf{q}^{\prime}\right) \quad \mathbf{q}\right] \mathbf{q}=[\Xi(\mathbf{q}) \quad \mathbf{q}] \mathbf{q}^{\prime}
$$

Also, the inverse quaternion is defined by

$$
\mathbf{q}^{-1}=\left[\begin{array}{c}
-\boldsymbol{\rho} \\
q_{4}
\end{array}\right]
$$

Note that $\mathbf{q} \otimes \mathbf{q}^{-1}=\left[\begin{array}{llll}0 & 0 & 0 & 1\end{array}\right]^{T}$, which is the identity quaternion. 


\section{ECEF Frame Navigation Equations}

In this section the $E$ frame navigation equations with the quaternion parameterization are derived. Beginning from Newton's $2^{\text {nd }}$ law of motion in the gravitational field of the Earth, which relates specific force and gravitation, the total acceleration is given by

$$
\frac{d^{2} \mathbf{r}_{e b}}{d t^{2}}=\mathbf{f}_{i b}+\gamma_{i b}
$$

where $\mathbf{r}_{e b}$ is the position vector of the body relative to the $E$ frame, $\mathbf{f}_{i b}$ is the specific force vector sensed with respect to the $I$ frame, and $\gamma_{i b}$ is the gravitation which does not incorporate with any centripetal components. The translational velocity is obtained by taking time derivative of the position vector $\mathbf{r}_{e b}$ :

$$
\begin{gathered}
\left.\frac{d \mathbf{r}_{e b}}{d t}\right|_{I}=\left.\frac{d \mathbf{r}_{e b}}{d t}\right|_{E}+\left[\boldsymbol{\omega}_{i e} \times\right] \mathbf{r}_{e b} \\
\text { with } \mathbf{v}_{e b}^{e}=\left.\frac{d \mathbf{r}_{e b}}{d t}\right|_{E}
\end{gathered}
$$

where $\mathbf{v}_{e b}^{e}$ is the velocity of the body relative to the $E$ frame described in the $E$ frame.

Equation (12) is then given by

$$
\left.\frac{d \mathbf{r}_{e b}}{d t}\right|_{I}=\mathbf{v}_{e b}^{e}+\left[\boldsymbol{\omega}_{i e} \times\right] \mathbf{r}_{e b}
$$

Time derivative of Eq. (14) yields the equations of the translational motion:

$$
\begin{aligned}
\left.\frac{d^{2} \mathbf{r}_{e b}}{d t^{2}}\right|_{I} & =\left.\frac{d \mathbf{v}_{e b}}{d t}\right|_{E}+\left[\boldsymbol{\omega}_{i e} \times\right] \mathbf{v}_{e b}+\left[\left.\frac{d \boldsymbol{\omega}_{i e}}{d t}\right|_{E} \times\right] \mathbf{r}_{e b}+\left[\boldsymbol{\omega}_{i e} \times\right]\left(\left.\frac{d \mathbf{r}_{e b}}{d t}\right|_{E}+\left[\boldsymbol{\omega}_{i e} \times\right] \mathbf{r}_{e b}\right) \\
& =\left.\frac{d \mathbf{v}_{e b}}{d t}\right|_{E}+2\left[\boldsymbol{\omega}_{i e} \times\right] \mathbf{v}_{e b}+\left[\left.\frac{d \boldsymbol{\omega}_{i e}}{d t}\right|_{E} \times\right] \mathbf{r}_{e b}+\left[\boldsymbol{\omega}_{i e} \times\right]\left[\boldsymbol{\omega}_{i e} \times\right] \mathbf{r}_{e b}
\end{aligned}
$$

Substituting Eq. (11) into Eq. (15) yields

$$
\left.\frac{d \mathbf{v}_{e b}}{d t}\right|_{E}=\mathbf{f}-2 \boldsymbol{\omega}_{i e} \times \mathbf{v}_{e b}-\boldsymbol{\omega}_{i e} \times \boldsymbol{\omega}_{i e} \times \mathbf{r}_{e b}+\boldsymbol{\gamma}_{i b}
$$

Representing Eq. (13) and (16) resolved about the $E$ frame is given by

$$
\begin{gathered}
\dot{\mathbf{r}}_{e b}^{e}=\mathbf{v}_{e b}^{e} \\
\dot{\mathbf{v}}_{e b}^{e}=C_{b}^{e} \mathbf{f}_{i b}^{b}-2 \Omega_{i e}^{e} \mathbf{v}_{e b}^{e}-\Omega_{i e}^{e 2} \mathbf{r}_{e b}^{e}+\gamma_{e b}^{e}
\end{gathered}
$$

where $\mathbf{r}_{e b}^{e}=\left[\begin{array}{lll}x_{e} & y_{e} & z_{e}\end{array}\right]^{T}, \Omega_{i e}^{e}=\left[\boldsymbol{\omega}_{i e}^{e} \times\right], \quad \boldsymbol{\omega}_{i e}^{e}=\left[\begin{array}{lll}0 & 0 & \omega_{i e}\end{array}\right]^{T}, \omega_{i e}$ is the Earth's angular rate, $\mathbf{f}_{i b}^{b}$ is the specific force vector relative to the $I$ frame resolved about the $B$ frame and $\gamma_{e b}^{e}=\left[\begin{array}{lll}\gamma_{x} & \gamma_{y} & \gamma_{z}\end{array}\right]^{T}$ is the gravitational force vector of the body in the $E$ frame. The components of the gravitational force vector in the $E$ frame considering up to $2^{\text {nd }}$ order polynomial are given by 


$$
\begin{aligned}
& \gamma_{x}=-\frac{\mu}{R^{2}}\left[1-\frac{3}{2} J_{2}\left(\frac{R_{e}}{R}\right)^{2}\left(5 \frac{z_{e}^{2}}{R^{2}}-1\right)\right] \frac{x_{e}}{R} \\
& \gamma_{y}=-\frac{\mu}{R^{2}}\left[1-\frac{3}{2} J_{2}\left(\frac{R_{e}}{R}\right)^{2}\left(5 \frac{z_{e}^{2}}{R^{2}}-1\right)\right] \frac{y_{e}}{R} \\
& \gamma_{z}=-\frac{\mu}{R^{2}}\left[1-\frac{3}{2} J_{2}\left(\frac{R_{e}}{R}\right)^{2}\left(5 \frac{z_{e}^{2}}{R^{2}}-3\right)\right] \frac{z_{e}}{R}
\end{aligned}
$$

where $R=\sqrt{x_{e}^{2}+y_{e}^{2}+z_{e}^{2}}$ is the range from the center of the Earth to the center of mass of the body, $J_{2}$ is the coefficient of second zonal harmonics of the Earth potential. Combining Eqs. (6), (17) and (18) yields the nonlinear mechanization equations in the $E$ frame.

$$
\left[\begin{array}{c}
\dot{\mathbf{q}} \\
\dot{\mathbf{r}}_{e b}^{e} \\
\dot{\mathbf{v}}_{e b}^{e}
\end{array}\right]=\left[\begin{array}{c}
\frac{1}{2} \Xi(\mathbf{q}) \boldsymbol{\omega}_{e b}^{e} \\
\mathbf{v}_{e b}^{e} \\
C_{b}^{e} \mathbf{f}_{i b}^{b}-2 \Omega_{i e}^{e} \mathbf{v}_{e b}^{e}-\left(\Omega_{i e}^{e}\right)^{2} \mathbf{r}_{e b}^{e}+\boldsymbol{\gamma}_{e b}^{e}
\end{array}\right]
$$

Figure 1 shows the algorithmic flowchart of the mechanization equation in the $E$ frame. In this algorithm, the numerical integration of the IMU raw data is computed in two steps. In the first step, the quaternion kinematics including the angular velocity $\boldsymbol{\omega}_{i b}^{b}$ is integrated numerically to obtain the transformation matrix from the $E$ to the $B$ frame. In the second step, the specific force $\mathbf{f}_{i b}^{b}$ is transformed into the $E$ frame, and then the velocity and position of the body are obtained by integrating the acceleration in the $E$ frame consecutively. To represent the attitude in the $N$ frame the rotation matrix $C_{n}^{b}$ from the $N$ frame to the $B$ frame is obtained through the successive rotation matrices:

$$
\begin{gathered}
C_{n}^{b}=C_{e}^{b}\left(C_{e}^{n}\right)^{T} \\
C_{e}^{n}=\left[\begin{array}{ccc}
-\sin \Phi \cos \Lambda & -\sin \Phi \sin \Lambda & \cos \Phi \\
-\sin \Lambda & \cos \Lambda & 0 \\
-\cos \Phi \cos \Lambda & -\cos \Phi \sin \Lambda & -\sin \Phi
\end{array}\right]
\end{gathered}
$$

where $C_{e}^{n}$ is the rotation matrix that transforms the $E$ frame to the $N$ frame, $\Phi$ and $\Lambda$ are geodetic latitude and longitude which can be computed using the position vector $\mathbf{r}_{e b}^{e}$ with WGS-84 parameters [14,15].

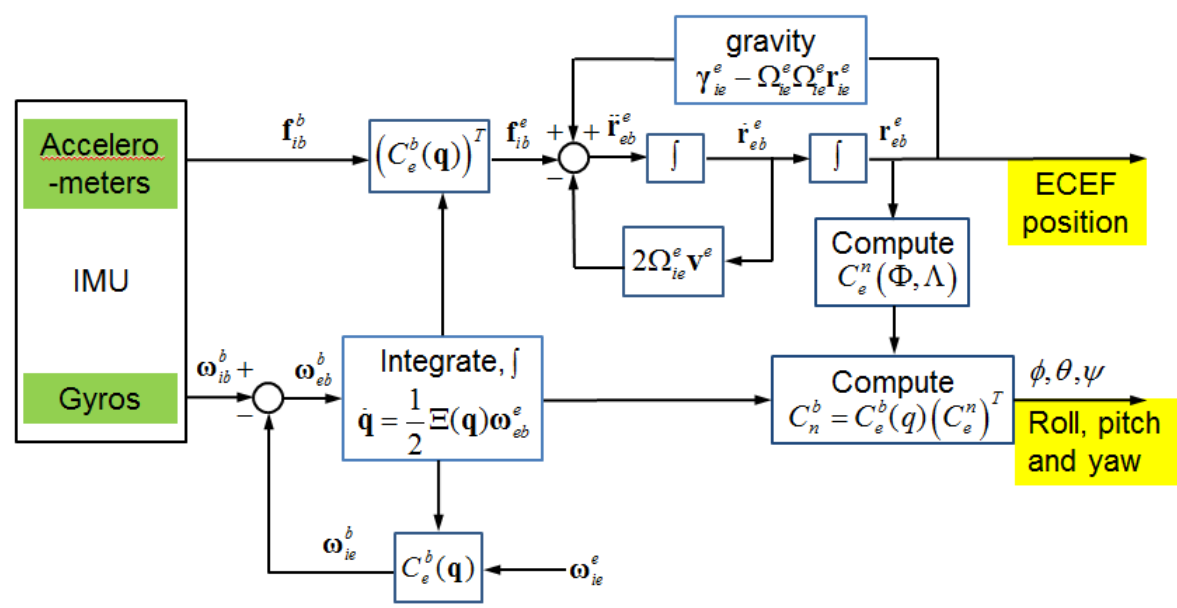

Figure 1. Algorithmic flowchart mechanization equation in the ECEF frame. 
Here the gyro measurement model is given by [4]

$$
\begin{gathered}
\tilde{\boldsymbol{\omega}}_{i b}^{b}=\left(I_{3 \times 3}+S_{g}\right) \boldsymbol{\omega}_{i b}^{b}+\boldsymbol{\beta}_{g}+\boldsymbol{\eta}_{g v} \\
\dot{\boldsymbol{\beta}}_{g}=\boldsymbol{\eta}_{g u}
\end{gathered}
$$

where $\boldsymbol{\omega}_{i b}^{b}$ is the true angular velocity of the $B$ frame relative to $I$ frame expressed in the $B$ coordinates, $\tilde{\boldsymbol{\omega}}_{i b}^{b}$ is the measured angular velocity of the gyro, $\boldsymbol{\beta}_{g}$ is the gyro bias, $S_{g}$ is a diagonal matrix of gyro scale factors, and $\boldsymbol{\eta}_{g v}$ and $\boldsymbol{\eta}_{g u}$ are zero-mean Gaussian white-noise process with spectral densities given by $\sigma_{a v}^{2} I_{3 \times 3}$ and $\sigma_{a u}^{2} I_{3 \times 3}$, respectively. The accelerometer measurement model is given by [4]

$$
\begin{gathered}
\tilde{\mathbf{f}}_{i b}^{b}=\left(I_{3 \times 3}+S_{a}\right) \mathbf{f}_{i b}^{b}+\boldsymbol{\beta}_{a}+\boldsymbol{\eta}_{a v} \\
\dot{\boldsymbol{\beta}}_{a}=\boldsymbol{\eta}_{a u}
\end{gathered}
$$

where $\mathbf{f}_{i b}^{b}$ is the true specific force of the $B$ frame relative to $I$ frame expressed in the $B$ coordinates, $\tilde{\mathbf{f}}_{i b}^{b}$ is the measured specific force of the accelerometer, $\boldsymbol{\beta}_{a}$ the accelerometer bias, $S_{a}$ is a diagonal matrix of accelerometer scale factors, $\boldsymbol{\eta}_{a v}$ and $\boldsymbol{\eta}_{a u}$ are zero-mean Gaussian white-noise process with spectral densities given by $\sigma_{a v}^{2} I_{3 \times 3}$ and $\sigma_{a u}^{2} I_{3 \times 3}$, respectively. The scale factors are assumed to be small enough so that the approximation $\left(I_{3 \times 3}+S\right)^{-1} \approx\left(I_{3 \times 3}-S\right)$ is valid for both the gyros and accelerometers, which will be used in the EKF derivation [1].

\section{Extended Kalman Filter equations for loosely coupled GPS/INS integration}

In this section the implementation equations for the EKF for loosely coupled GPS/INS integration using the ECEF frame are shown. The GPS navigation solutions are used as measurements to the EKF, which are used to correct the INS errors. Here the GPS navigation solutions are assumed to be available through the GPS Kalman filter navigation in the GPS receiver and have two possible GPS navigation solutions. One is the position of a vehicle in the ECEF frame and the other is both the position and velocity a vehicle in the ECEF frame. The estimated quantities are given by $[1,3]$

$$
\begin{gathered}
\dot{\hat{\mathbf{q}}}=\frac{1}{2} \Xi(\hat{\mathbf{q}}) \hat{\boldsymbol{\omega}}_{e b}^{e} \\
\hat{\boldsymbol{\omega}}_{e b}^{b}=\left(I_{3 \times 3}-\hat{S}_{g}\right)\left(\tilde{\boldsymbol{\omega}}_{i b}^{b}-\hat{\boldsymbol{\beta}}_{g}\right)-C_{E}^{B}(\hat{\mathbf{q}}) \boldsymbol{\omega}_{i e}^{e} \\
\dot{\hat{\mathbf{r}}}_{e b}^{e}=\hat{\mathbf{v}}_{e b}^{e} \\
\dot{\hat{\mathbf{v}}}_{i e}^{e}=C_{b}^{e}(\hat{\mathbf{q}}) \hat{\mathbf{f}}_{i b}^{b}-2 \Omega_{i e}^{e} \hat{\mathbf{v}}_{i e}^{e}-\Omega_{i e}^{e 2} \hat{\mathbf{r}}_{i e}^{e}+\hat{\boldsymbol{\gamma}}_{i e}^{e}\left(\hat{\mathbf{r}}_{i e}^{e}\right) \\
\hat{\mathbf{f}}_{i b}^{b}=\left(I_{3 \times 3}-\hat{S}_{a}\right)\left(\tilde{\mathbf{f}}_{i b}^{b}-\hat{\boldsymbol{\beta}}_{a}\right) \\
\dot{\hat{\boldsymbol{\beta}}}_{g}=\mathbf{0} \\
\dot{\hat{\boldsymbol{\beta}}}_{a}=\mathbf{0} \\
\dot{\hat{\mathbf{S}}}_{g}=\mathbf{0} \\
\dot{\hat{\mathbf{s}}}_{a}=\mathbf{0}
\end{gathered}
$$


where $\mathbf{0}$ is a $3 \times 1$ null matrix, $\hat{\mathbf{s}}_{g}$ and $\hat{\mathbf{s}}_{a}$ the elements of the diagonal matrices $\hat{S}_{g}$ and $\hat{S}_{a}$, respectively. Also, $C_{b}^{e}(\hat{\mathbf{q}}), \hat{\mathbf{f}}_{i b}^{b}$ and $\hat{\boldsymbol{\gamma}}_{i e}^{e}\left(\hat{\mathbf{r}}_{i e}^{e}\right)$ are evaluated at the present estimates. The attitude matrix $C_{b}^{e}(\hat{\mathbf{q}})$ is computed using Eq. (1). Note that the attitude matrix $C_{b}^{e}(\hat{\mathbf{q}})$ of Eq. (28) is coupled into the position vector as shown in Eq. (28), which permits to estimate the attitude from the position measurements.

We now derive the attitude error equations, which are used in the EKF covariance propagation. The linearized model error kinematics follows directly from that the quaternion is linearized using a multiplicative approach $[3,13]$. The error quaternion and its derivatives are given by

$$
\delta \mathbf{q}=\mathbf{q} \otimes \hat{\mathbf{q}}^{-1}
$$

with $\delta \mathbf{q} \equiv\left[\begin{array}{ll}\delta \boldsymbol{\rho}^{T} & \delta q_{4}\end{array}\right]^{T}$, where the quaternion multiplication is defined by Eq. (9). The equivalent attitude error matrix is given by

$$
C_{E}^{B}(\delta \mathbf{q})=C_{E}^{B}(\mathbf{q})\left[C_{E}^{B}(\hat{\mathbf{q}})\right]^{T}
$$

If the error quaternion is small enough to within first order, the vector part of the quaternion error can be approximated by $\delta \boldsymbol{\rho} \approx \delta \boldsymbol{\alpha} / 2$ and $\delta q_{4} \approx 1$, where $\delta \boldsymbol{\alpha}$ is a small error-angle rotation vector. Also, the quaternion inverse is defined by Eq. (10). The linearized model error-kinematics then takes the form:

$$
\begin{gathered}
\delta \dot{\boldsymbol{\alpha}}=-\left[\hat{\boldsymbol{\omega}}_{i b}^{b}\right]^{\times} \delta \boldsymbol{\alpha}+\delta \boldsymbol{\omega}_{i b}^{b}-C_{E}^{B}\left(\hat{\mathbf{q}}^{e}\right) \delta \boldsymbol{\omega}_{i e}^{e} \\
\delta \dot{q}_{4}=0
\end{gathered}
$$

where $\delta \boldsymbol{\omega}_{i b}^{b}=\boldsymbol{\omega}_{i b}^{b}-\hat{\boldsymbol{\omega}}_{i b}^{b}$ and $\delta \boldsymbol{\omega}_{i e}^{e}=\mathbf{0}$ since $\boldsymbol{\omega}_{i e}^{e}$ is constant. Note that the fourth error-quaternion component is constant. The first-order approximation, which assumes that the true quaternion is close to the estimated quaternion, yields $\delta q_{4} \approx 1$. The order of the system in the EKF is then reduced to one state using this property. From the angular velocity model of Eq. (23a), the error of $\delta \boldsymbol{\omega}_{i b}^{b}$ to within first-order approximation in the EKF can be written using the approximation $\left(I_{3 \times 3}+S_{g}\right)^{-1} \approx\left(I_{3 \times 3}-S_{g}\right)[1]$ as

$$
\begin{aligned}
\delta \boldsymbol{\omega}_{i b}^{b} & =\left(I_{3 \times 3}-S_{g}\right)\left(\tilde{\boldsymbol{\omega}}_{i b}^{b}-\boldsymbol{\beta}_{g}-\boldsymbol{\eta}_{g v}\right)-\left(I_{3 \times 3}-\hat{S}_{g}\right)\left(\tilde{\boldsymbol{\omega}}_{i b}^{b}-\hat{\boldsymbol{\beta}}_{g}\right) \\
& =-\left[\left(I_{3 \times 3}-S_{g}\right) \Delta \boldsymbol{\beta}_{g}+\left(\tilde{\Omega}_{i b}^{b}-\hat{B}_{g}\right) \Delta \mathbf{s}_{g}+\left(I_{3 \times 3}-S_{g}\right) \boldsymbol{\eta}_{g v}\right]
\end{aligned}
$$

where $\Delta \boldsymbol{\beta}_{g}=\boldsymbol{\beta}_{g}-\hat{\boldsymbol{\beta}}_{g}, \Delta \mathbf{s}_{g}=\mathbf{s}_{g}-\hat{\mathbf{s}}_{g}, \tilde{\Omega}_{i b}^{b}$ is a diagonal matrix of the elements of $\tilde{\boldsymbol{\omega}}_{i b}^{b}$ and $\hat{B}_{g}$ is the a diagonal matrix of the elements of $\hat{\boldsymbol{\beta}}_{g}$. The error $\delta \boldsymbol{\omega}_{i e}^{e}$ can be computed using a first-order Taylor series expansion. This yields

$$
\delta \dot{\boldsymbol{\alpha}}=-\left[\left(I_{3 \times 3}-\hat{S}_{g}\right)\left(\tilde{\boldsymbol{\omega}}_{i b}^{b}-\hat{\boldsymbol{\beta}}_{g}\right) \times\right] \delta \boldsymbol{\alpha}-\left(I_{3 \times 3}-S_{g}\right) \Delta \boldsymbol{\beta}_{g}-\left(\tilde{\Omega}_{i b}^{b}-\hat{B}_{g}\right) \Delta \mathbf{s}_{g}-\left(I_{3 \times 3}-S_{g}\right) \boldsymbol{\eta}_{g v}
$$

The state, state-error vector, process noise vector and covariance used in the EKF are defined as 


$$
\begin{gathered}
\mathbf{x} \equiv\left[\begin{array}{c}
\mathbf{q} \\
\mathbf{r}_{e b}^{e} \\
\mathbf{v}_{e b}^{e} \\
\boldsymbol{\beta}_{g} \\
\boldsymbol{\beta}_{g} \\
\mathbf{s}_{g} \\
\mathbf{s}_{a}
\end{array}\right], \quad \Delta \mathbf{x} \equiv\left[\begin{array}{c}
\delta \boldsymbol{\alpha} \\
\Delta \mathbf{r}_{e b}^{e} \\
\Delta \mathbf{v}_{e b}^{e} \\
\Delta \boldsymbol{\beta}_{g} \\
\Delta \boldsymbol{\beta}_{g} \\
\Delta \mathbf{s}_{g} \\
\Delta \mathbf{s}_{a}
\end{array}\right], \quad \mathbf{w}=\left[\begin{array}{c}
\boldsymbol{\eta}_{g v} \\
\boldsymbol{\eta}_{g u} \\
\boldsymbol{\eta}_{a v} \\
\boldsymbol{\eta}_{a u}
\end{array}\right] \\
Q=\left[\begin{array}{cccc}
\sigma_{g v}^{2} I_{3 \times 3} & 0_{3 \times 3} & 0_{3 \times 3} & 0_{3 \times 3} \\
0_{3 \times 3} & \sigma_{g u}^{2} I_{3 \times 3} & 0_{3 \times 3} & 0_{3 \times 3} \\
0_{3 \times 3} & 0_{3 \times 3} & \sigma_{a v}^{2} I_{3 \times 3} & 0_{3 \times 3} \\
0_{3 \times 3} & 0_{3 \times 3} & 0_{3 \times 3} & \sigma_{a u}^{2} I_{3 \times 3}
\end{array}\right]
\end{gathered}
$$

where $0_{3 \times 3}$ is a $3 \times 3$ null matrix, the state vector $\mathbf{x}$ is a 22 -dimensional vector, and the state-error vector $\Delta \mathbf{x}$ is a 21-dimensional vector since a 4-dimensional quaternion is used to describe three dimensions. The error-dynamics used in the EKF are given by

$$
\begin{gathered}
\Delta \dot{\mathbf{x}}=F \Delta \mathbf{x}+G \mathbf{w} \\
F=\left[\begin{array}{ccccccc}
F_{11} & 0_{3 \times 3} & 0_{3 \times 3} & F_{14} & 0_{3 \times 3} & F_{16} & 0_{3 \times 3} \\
0_{3 \times 3} & 0_{3 \times 3} & I_{3 \times 3} & 0_{3 \times 3} & 0_{3 \times 3} & 0_{3 \times 3} & 0_{3 \times 3} \\
F_{31} & F_{32} & F_{33} & F_{34} & F_{35} & F_{36} & F_{37} \\
0_{3 \times 3} & 0_{3 \times 3} & 0_{3 \times 3} & 0_{3 \times 3} & 0_{3 \times 3} & 0_{3 \times 3} & 0_{3 \times 3} \\
0_{3 \times 3} & 0_{3 \times 3} & 0_{3 \times 3} & 0_{3 \times 3} & 0_{3 \times 3} & 0_{3 \times 3} & 0_{3 \times 3} \\
0_{3 \times 3} & 0_{3 \times 3} & 0_{3 \times 3} & 0_{3 \times 3} & 0_{3 \times 3} & 0_{3 \times 3} & 0_{3 \times 3} \\
0_{3 \times 3} & 0_{3 \times 3} & 0_{3 \times 3} & 0_{3 \times 3} & 0_{3 \times 3} & 0_{3 \times 3} & 0_{3 \times 3}
\end{array}\right] \\
G=\left[\begin{array}{cccccc}
-\left(I_{3 \times 3}-\hat{S}_{g}\right) & 0_{3 \times 3} & 0_{3 \times 3} & 0_{3 \times 3} \\
0_{3 \times 3} & 0_{3 \times 3} & 0_{3 \times 3} & 0_{3 \times 3} \\
0_{3 \times 3} & 0_{3 \times 3} & -C_{B}^{E}\left(\hat{\mathbf{q}}^{e}\right)\left(I_{3 \times 3}-\hat{S}_{g}\right) & 0_{3 \times 3} \\
0_{3 \times 3} & I_{3 \times 3} & 0_{3 \times 3} & 0_{3 \times 3} \\
0_{3 \times 3} & 0_{3 \times 3} & 0_{3 \times 3} & I_{3 \times 3} \\
0_{3 \times 3} & 0_{3 \times 3} & 0_{3 \times 3} & 0_{3 \times 3} \\
0_{3 \times 3} & 0_{3 \times 3} & 0_{3 \times 3} & 0_{3 \times 3}
\end{array}\right]
\end{gathered}
$$

with

$$
F_{11}=-\left[\left(I_{3 \times 3}-\hat{S}_{g}\right)\left(\tilde{\boldsymbol{\omega}}_{i b}^{b}-\hat{\boldsymbol{\beta}}_{g}\right) \times\right], F_{14}=-\left(I_{3 \times 3}-\hat{S}_{g}\right), F_{16}=-\left(\tilde{\Omega}_{i b}^{b}-\hat{B}_{g}\right)
$$

$$
\begin{gathered}
F_{31}=-C_{B}^{E}\left(\hat{\mathbf{q}}^{e}\right)\left[\hat{\mathbf{f}}_{i b}^{b} \times\right], F_{32}=-\left(\Omega_{i e}^{e}\right)^{2}+\left.\nabla \hat{\boldsymbol{\gamma}}\right|_{\hat{r}_{e b}^{e}}, F_{33}=-2 \Omega_{i e}^{e} \\
F_{35}=-C_{B}^{E}\left(\hat{\mathbf{q}}^{e}\right)\left(I_{3 \times 3}-\hat{S}_{a}\right), F_{37}=-C_{B}^{E}\left(\hat{\mathbf{q}}^{e}\right)\left(\tilde{F}_{i b}^{b}-\hat{S}_{a}\right)
\end{gathered}
$$

where $\tilde{F}_{i b}^{b}$ is a diagonal matrix of the elements of $\tilde{\mathbf{f}}_{i b}^{b}$ and $\hat{S}_{a}$ is a diagonal matrix of the elements of $\hat{\mathbf{s}}_{a}$. More details of Eq. (44) derivations are explained in Appendix.

The EKF algorithm for loosely coupled GPS/INS integration is summarized in Table 1. The EKF is composed of initialization, propagation, gain computation and update. The GPS navigation solutions are used to the EKF measurements and are assumed to be modeled as

$$
\tilde{\mathbf{r}}_{e b, k}^{e}=\mathbf{r}_{e b, k}^{e}+\boldsymbol{\eta}_{r}
$$




$$
\tilde{\mathbf{v}}_{e b, k}^{e}=\mathbf{v}_{e b, k}^{e}+\boldsymbol{\eta}_{v}
$$

where $\boldsymbol{\eta}_{r}$ and $\boldsymbol{\eta}_{v}$ are zero-mean Gaussian noise processes with spectral densities given by $\sigma_{r}^{2} I_{3 \times 3}$ and $\sigma_{v}^{2} I_{3 \times 3}$, respectively.

Table 1. Extended Kalman filter for loosely coupled GPS/INS integration

\begin{tabular}{|c|c|}
\hline Initialization & $\hat{\mathbf{x}}_{k-1}=\hat{\mathbf{x}}_{0}, P_{k-1}=P_{0}, R_{k}, \quad \Phi\left(t_{k-1}, t_{0}\right)=\Phi\left(t_{0}, t_{0}\right)=I_{21 \times 21}$ \\
\hline Propagation & $\begin{array}{c}\hat{\boldsymbol{\omega}}_{e b}^{b}=\left(I_{3 \times 3}-\hat{S}_{g}\right)\left(\tilde{\boldsymbol{\omega}}_{i b}^{b}-\hat{\boldsymbol{\beta}}_{g}\right)-C_{E}^{B}\left(\hat{\mathbf{q}}^{e}\right) \boldsymbol{\omega}_{i e}^{e} \\
\dot{\hat{\mathbf{q}}}=\frac{1}{2} \Xi(\hat{\mathbf{q}}) \hat{\boldsymbol{\omega}}_{e b}^{e} \\
\hat{\mathbf{f}}_{i b}^{b}=\left(I_{3 \times 3}-\hat{S}_{a}\right)\left(\tilde{\mathbf{f}}_{i b}^{b}-\hat{\boldsymbol{\beta}}_{a}\right) \\
\dot{\hat{\mathbf{r}}}_{e b}^{e}=\hat{\mathbf{v}}_{e b}^{e} \\
\dot{\hat{\mathbf{v}}}_{i e}^{e}=C_{b}^{e}(\hat{\mathbf{q}}) \hat{\mathbf{f}}_{i b}^{b}-2 \Omega_{i e}^{e} \hat{\mathbf{v}}_{i e}^{e}-\Omega_{i e}^{e 2} \hat{\mathbf{r}}_{i e}^{e}+\hat{\boldsymbol{\gamma}}_{i e}^{e}\left(\hat{\mathbf{r}}_{i e}^{e}\right) \\
\dot{\Phi}\left(t_{k}, t_{k-1}\right)=F(t) \Phi\left(t_{k}, t_{k-1}\right) \\
\bar{P}_{k}=\Phi\left(t_{k}, t_{k-1}\right) P_{k-1}^{-} \Phi^{T}\left(t_{k}, t_{k-1}\right)+\bar{Q}_{k-1}\end{array}$ \\
\hline Gain & 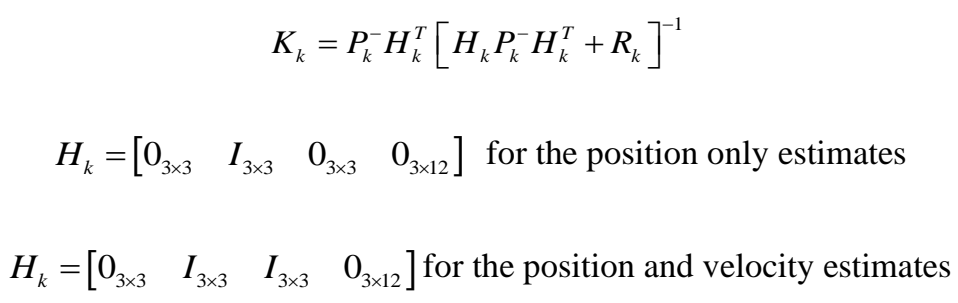 \\
\hline Update & $\begin{array}{c}\tilde{\mathbf{y}}_{k}=\tilde{\mathbf{r}}_{e b, k}^{e}, \tilde{\mathbf{y}}_{k}=\tilde{\mathbf{v}}_{e b, k}^{e} \text { or } \tilde{\mathbf{y}}_{k}=\left[\begin{array}{c}\tilde{\mathbf{r}}_{e b, k}^{e} \\
\tilde{\mathbf{v}}_{e b, k}^{e}\end{array}\right], \quad \hat{\mathbf{y}}_{k}=\hat{\mathbf{r}}_{e b, k}^{e}, \text { or } \hat{\mathbf{y}}_{k}=\left[\begin{array}{c}\hat{\mathbf{r}}_{e b, k}^{e} \\
\hat{\mathbf{v}}_{e b, k}^{e}\end{array}\right] \\
\Delta \mathbf{x}_{k}=K_{k}\left[\tilde{\mathbf{y}}_{k}-\hat{\mathbf{y}}_{k}\right] \\
P_{k}=\left[I_{21 \times 21}-K_{k} H_{k}\right] \bar{P}_{k} \\
\hat{\mathbf{q}}_{k}^{+}=\delta \hat{\mathbf{q}}_{k}^{+} \otimes \hat{\mathbf{q}}_{k}^{-}=\left[\begin{array}{c}\frac{1}{2} \delta \hat{\boldsymbol{\alpha}}_{k}^{+} \\
1\end{array}\right] \otimes \hat{\mathbf{q}}_{k}^{-}=\hat{\mathbf{q}}_{k}^{-}+\frac{1}{2} \Xi\left(\hat{\mathbf{q}}_{k}^{-}\right) \delta \hat{\boldsymbol{\alpha}}_{k}^{+}, \\
\text {renormalize quaternion }\end{array}$ \\
\hline
\end{tabular}




\begin{tabular}{l|l}
\hline & $\hat{\mathbf{r}}_{e b, k}^{e+}=\hat{\mathbf{r}}_{e b, k}^{e-}+\Delta \hat{\mathbf{r}}_{e b, k}^{e+}$ \\
& $\hat{\mathbf{v}}_{e b, k}^{e+}=\hat{\mathbf{v}}_{e b, k}^{e-}+\Delta \hat{\mathbf{v}}_{e b, k}^{e+}$ \\
& $\hat{\boldsymbol{\beta}}_{g, k}^{+}=\hat{\boldsymbol{\beta}}_{g, k}^{-}+\Delta \hat{\boldsymbol{\beta}}_{g, k}^{+}$ \\
& $\hat{\boldsymbol{\beta}}_{a, k}^{+}=\hat{\boldsymbol{\beta}}_{a, k}^{-}+\Delta \hat{\boldsymbol{\beta}}_{a, k}^{+}$ \\
& $\hat{\mathbf{s}}_{g, k}^{+}=\hat{\mathbf{s}}_{g, k}^{-}+\Delta \hat{\mathbf{s}}_{g, k}^{+}$ \\
& $\hat{\mathbf{s}}_{a, k}^{+}=\hat{\mathbf{s}}_{a, k}^{-}+\Delta \hat{\mathbf{s}}_{a, k}^{+}$ \\
\hline
\end{tabular}

The EKF is initialized with a known state (the bias initial conditions for the gyro and accelerometer are usually set to zero) and error covariance matrix. The first three diagonal elements of the error covariance matrix correspond to attitude errors of the body-fixed frame with respect to the ECEF frame Then, the Kalman gain is computed using the measurement error covariance matrix $R_{k}$ and sensitivity matrix $H_{k}$. The sensitivity matrix $H_{k}$ for the position, and both the position and velocity are given by

$$
\begin{aligned}
& H_{k}=\left[\begin{array}{llll}
0_{3 \times 3} & I_{3 \times 3} & 0_{3 \times 3} & 0_{3 \times 12}
\end{array}\right] \\
& H_{k}=\left[\begin{array}{llll}
0_{3 \times 3} & I_{3 \times 3} & I_{3 \times 3} & 0_{3 \times 12}
\end{array}\right]
\end{aligned}
$$

Thus, two different measurement types composed of the GPS position only, the GPS position and velocity can be considered in the EKF design. In general,

The corresponding measurement error covariance $R_{k}$ is given by

$$
\begin{gathered}
R_{k}=\sigma_{r}^{2} I_{3 \times 3} \\
R_{k}=\sigma_{v}^{2} I_{3 \times 3} \\
R_{k}=\left[\begin{array}{cc}
\sigma_{r}^{2} I_{3 \times 3} & 0_{3 \times 3} \\
0_{3 \times 3} & \sigma_{v}^{2} I_{3 \times 3}
\end{array}\right]
\end{gathered}
$$

The state error covariance follows the standard EKF update. The position, velocity, and bias states also follow the standard EKF additive correction while the attitude error state update is computed using a multiplicative update $[3,15]$. The updated quaternion is renormalized by brute force. Finally, the propagation equations follow the standard EKF model. To reduce the computational load a discrete-time propagation of the covariance matrix is used

$$
P_{k+1}^{-}=\Phi_{k} P_{k}^{-} \Phi_{k}^{T}+\bar{Q}_{k}
$$

where $\Phi_{k}$ is the discrete-time state transition matrix and $\bar{Q}_{k}$ is the discrete-time process noise covariance matrix. Though $\bar{Q}_{k}$ can be obtained using numerical solution given by van Loan [18], the first-order approximation of the solution is given by

$$
\bar{Q}_{k} \approx \Delta t G Q G^{T}
$$

where $\Delta t$ is the constant sampling time.

\section{Simulation results}

In this section simulation results are shown that estimate a flying vehicle's attitude, position, and velocity, as well as the gyro and accelerometer biases and scale factors. All measurements are assumed to be sampled 
every 0.1 seconds. The gyro measurements are simulated using Eqs. (23) with $\sigma_{g v}=\sqrt{10} \times 10^{-7} \mathrm{rad} / \mathrm{s}^{1 / 2}$ and $\sigma_{g u}=\sqrt{10} \times 10^{-10} \mathrm{rad} / \mathrm{s}^{3 / 2}$, and an initial bias $0.5 \mathrm{deg} / \mathrm{hr}$ on each axis. The accelerometer measurements are simulated using Eq. (24) with $\sigma_{a v}=9.81 \times 10^{-6} \mathrm{~m} / \mathrm{s}^{3 / 2}$ and $\sigma_{a u}=6 \times 10^{-5} \mathrm{~m} / \mathrm{s}^{5 / 2}$, and an initial bias $300 \mu g=2.9 \times 10^{-3} \mathrm{~m} / \mathrm{s}^{2}$ on each axis. Also, the scale factors are given by $S_{g}=10^{-2} I_{3 \times 3}$ and $S_{a}=10^{-4} I_{3 \times 3}$.

Initially the GPS navigation solution is simulated using Eqs. (45) and (46) with $\sigma_{r}=5 \mathrm{~m}$ and $\sigma_{v}=3 \times 10^{-2} \mathrm{~m} / \mathrm{s}$. The origin location of the aerial vehicle motion is described at $\Phi_{0}=35.139968$ degrees and $\Lambda_{0}=126.931658$ degrees. The initial quaternion is given so that the aerial vehicle body-fixed frame $B$ coincides with the local navigation frame $N$. The initial velocity is given by $\mathbf{v}^{n}=\left[\begin{array}{lll}0 & 2 & 0\end{array}\right]^{T} \mathrm{~m} / \mathrm{s}$. The rotational rate profile is given by: $5 \mathrm{deg} / \mathrm{min}$ rotation about the $x_{b}$ axis for the first $200 \mathrm{~s}$ and then zero for the final $400 \mathrm{~s}$; no rotation about the $y_{b}$ axis for the first $200 \mathrm{~s}$, then a $5 \mathrm{deg} / \mathrm{min}$ rotation for the next $200 \mathrm{~s}$ and zero for the final $400 \mathrm{~s}$; no rotation about the $z_{b}$ axis for the first $200 \mathrm{~s}$, then $5 \mathrm{deg} / \mathrm{min}$ rotation for the final $400 \mathrm{~s}$.

In the EKF an initial attitude error of 3,-3 and 3 degrees is given in each axis. The initial covariance matrix $P_{0}$ in the EKF is diagonal. For this case, the three attitude parts of the initial covariance are each set to a $3 \sigma$ bound of 5 degrees, i.e., $[(5 / 3) \times(\pi / 180)]^{2} \operatorname{rad}^{2}$. The initial estimates for position are set to the true position of the aerial vehicle in the $E$ frame adding the position error of $10 \mathrm{~m}$ in each axis. The initial variance for the position is given by $(10 / 3)^{2} \mathrm{~m}^{2}$. The initial velocity is set to the true velocity of the aerial vehicle in the $E$ frame adding the velocity error of $1 \mathrm{~m} / \mathrm{s}$ in each axis. The initial variance for the velocity is given by $(1 / 3)^{2} \mathrm{~m}^{2} / \mathrm{s}^{2}$. The initial gyro and accelerometer biases and scale factors are all set to zero. The three gyro-bias parts of the initial covariance are each set to a $3 \sigma$ bound of $3 \mathrm{deg} / \mathrm{hr}$, i.e., $[(3 / 3) \times(\pi(180 \times 3600))]^{2} \mathrm{rad}^{2} / \mathrm{s}^{2}$. The three accelerometer-bias parts of the initial covariance are each set to a $3 \sigma$ bound of $0.005 \mathrm{~m}$ per second-squared, i.e., $(0.005 / 3)^{2}$. The three gyro-scale factor parts of the initial covariance are each set to a $3 \sigma$ bound of 0.015 , i.e., $(0.015 / 3)^{2}$. Finally, the three accelerometer-scale factor parts of the initial covariance are each set to a $3 \sigma$ bound of 0.01 , i.e., $(0.01 / 3)^{2}$.

The resulting EKF using both the EKF position and velocity measurements are shown in Figure 2. Figure 2(a) shows an ascending flight trajectory in the $N$ frame, where the body-fixed frame of the aerial vehicle is plotted with respect to the $N$ frame along the trajectory. Figure 2 (b) shows the attitude estimation errors and respective $3 \sigma$ bounds derived from the EKF covariance matrix. The attitude errors are within their $3 \sigma$ bounds and converge to near zero at $3600 \mathrm{~s}$, which indicates that the EKF is performing in an optimal fashion. The large errors in pitch and yaw are due to the fact that these angles are the least observable state for the particular the aerial vehicle motion. Figure 3 (c) and (d) show the position and velocity estimation errors, and their $3 \sigma$ bounds, respectively. The position and velocity knowledges are within $1 \mathrm{~m}$ and $0.01 \mathrm{~m} / \mathrm{s}$ for each axis, respectively. The velocity estimation errors are shown for $60 \mathrm{~s}$ to observe fast convergence clearly, and they maintain their convergence for the rest of the simulation. Figure 2 (e) and (f) show the gyro and accelerometer bias estimation errors, and their respective $3 \sigma$ bounds. Figure $2(\mathrm{~g})$ and $(\mathrm{h})$ show the scale factor estimation errors of the gyro and accelerometer, and their $3 \sigma$ bounds, respectively. All errors remain within their respective bounds, which indicates that the EKF works properly. 


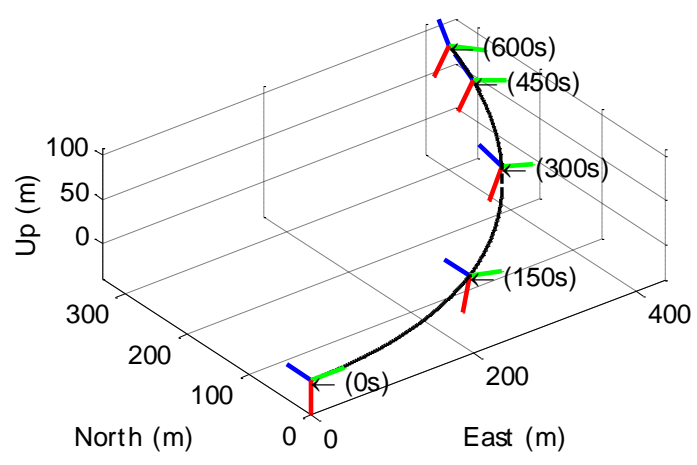

(a) Ascending flight trajectory in the ENU frame
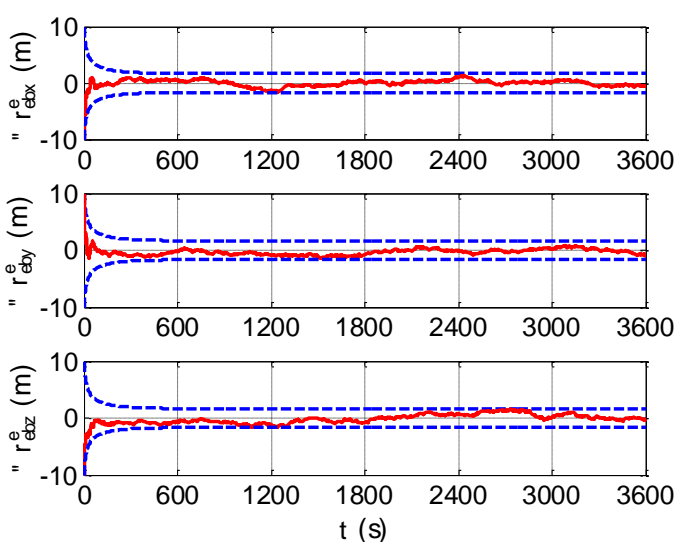

(c) Position estimation errors with $3 \sigma$ error bounds

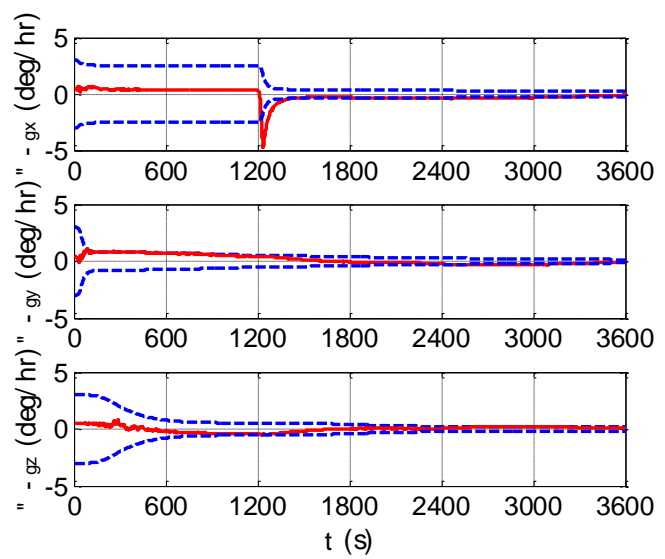

(e) Gyro bias estimation errors with $3 \sigma$ error bounds
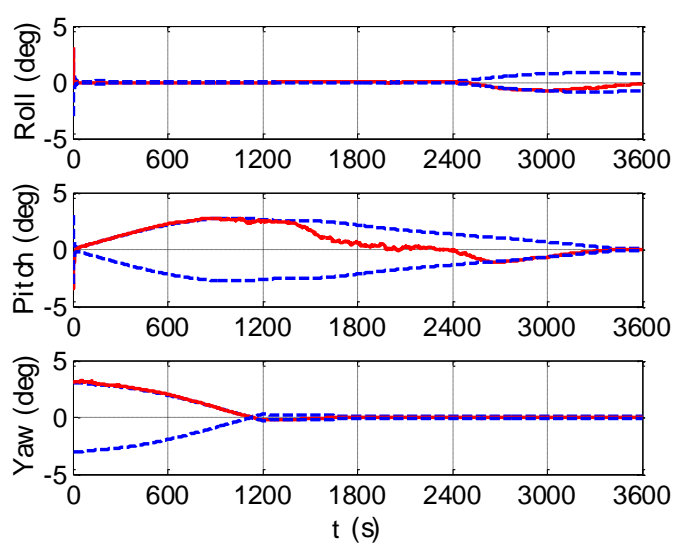

(b) Attitude estimation errors with $3 \sigma$ error bounds
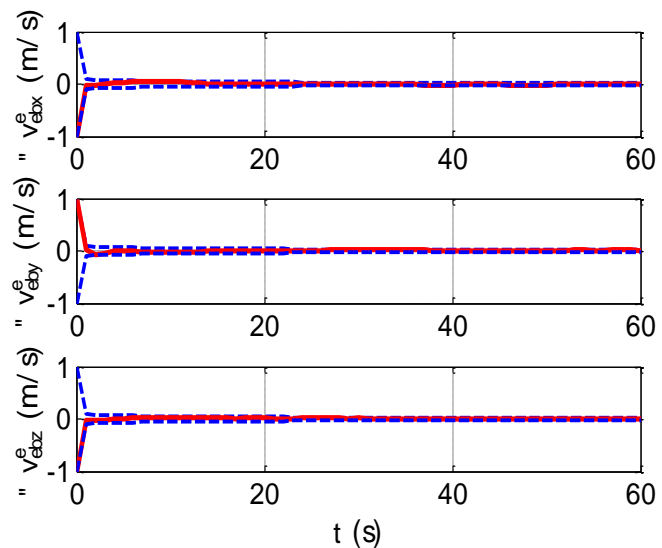

(d) Velocity estimation errors with $3 \sigma$ error bounds

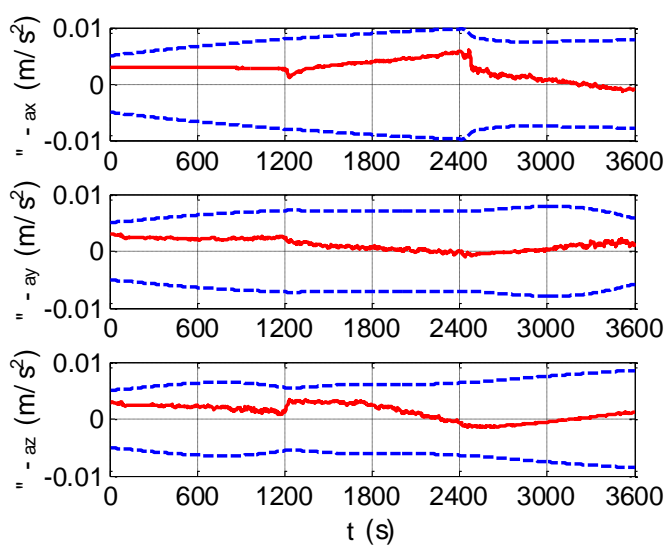

(f) Accelerometer bias estimation errors with $3 \sigma$ error bounds 

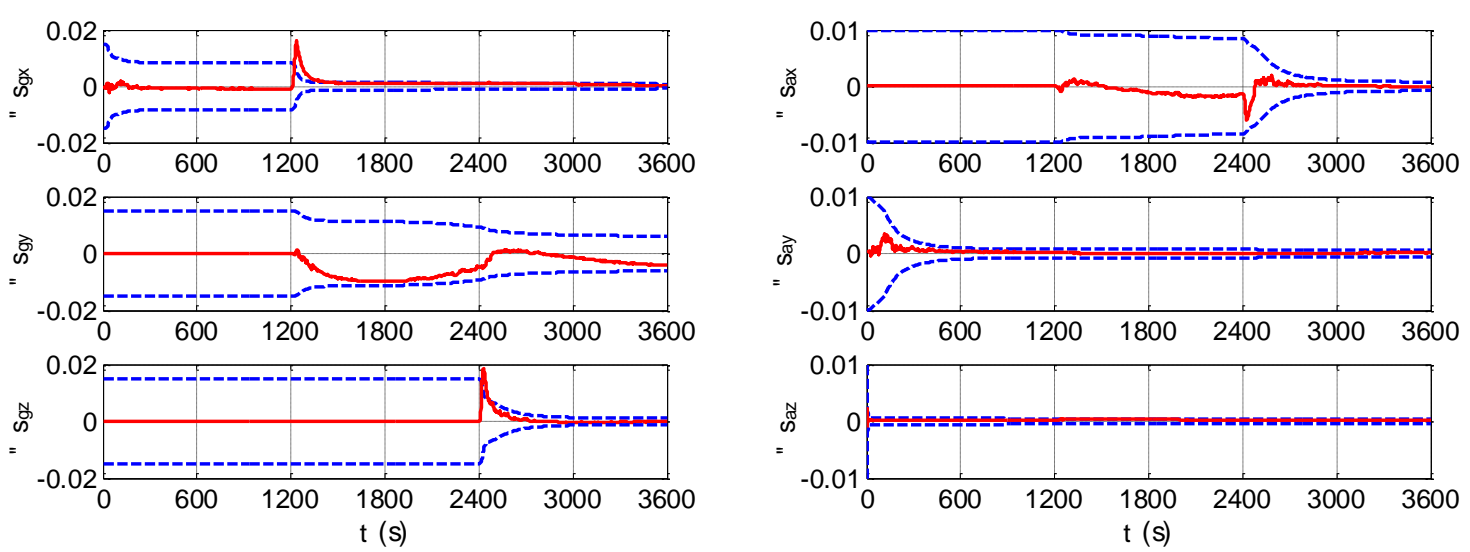

(g) Gyro scale factor estimation errors

(h) Accelerometer scale factor estimation with $3 \sigma$ error bounds errors with $3 \sigma$ error bounds

Figure 2. EKF estimation results for both the position and velocity measurements

To quantify the estimation results of the position and attitude, the norms of the attitude and position errors are numerically integrated, respectively over the $3600 \mathrm{~s}$ simulation, given by

$$
\begin{aligned}
& J_{a}=\int_{0}^{3600}\left\|\mathbf{e}_{a}(t)\right\| d t \\
& J_{r}=\int_{0}^{3600}\left\|\mathbf{e}_{r}(t)\right\| d t
\end{aligned}
$$

where $\mathbf{e}_{a}(t)$ denotes the roll, pitch and yaw errors in degrees and $\mathbf{e}_{r}(t)$ denotes the position estimation errors in meter. Then Eq. (38) is averaged over 30 runs (i.e, Monte-Carlo type simulation is performed). Table 2 shows the averaged $J_{a}$ and $J_{r}$ values for different measurements using both the position and velocity, and the velocity only, respectively. It shows that smaller averaged $J_{a}$ and $J_{r}$ are obtained when both the GPS position and velocity are used together.

Table 2. Averaged $J_{a}$ and $J_{r}$ values for different measurements

\begin{tabular}{c|c|c}
\hline Measurements & $J_{a}$ & $J_{r}$ \\
\hline Position and Velocity & $5.3430 \times 10^{3}$ & $1.0879 \times 10^{3}$ \\
\hline Position only & $5.4631 \times 10^{3}$ & $4.2788 \times 10^{3}$ \\
\hline
\end{tabular}

Figure 3 shows the norms of the position and attitude estimation errors using two different measurement combinations of the GPS navigation solutions, respectively. The norms of the attitude estimation errors are very similar and converge to less than 0.1 degrees in 3600 s, as shown in Fig. 2 (a). On the other hand, the norms of the position estimation errors converge to smaller than $1.2 \mathrm{~m}$ and $4 \mathrm{~m}$, respectively for both the position and velocity measurements, and the position velocity only. The values of $J_{a}$ and $J_{r}$ for the position and velocity measurements are smaller than the values of $J_{a}$ and $J_{r}$ for the position measurement only. More accurate EKF estimation result can be achieved when both the GPS position and velocity measurements are used, which indicates that the velocity measurements may improve the estimation performance with the position measurement. 


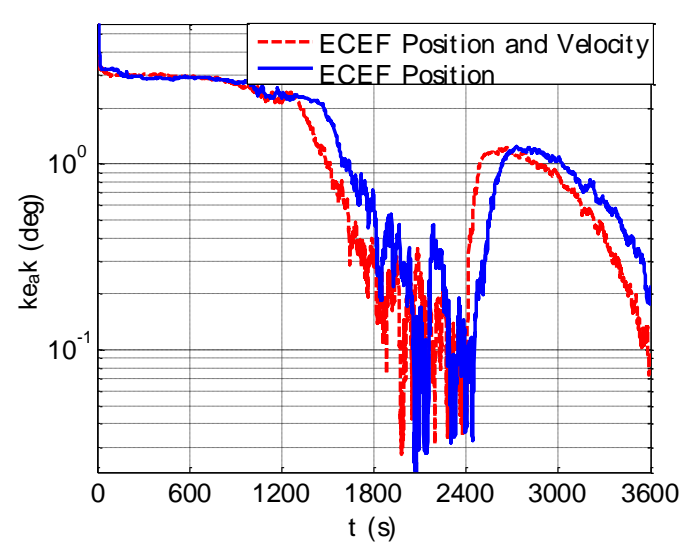

(a) Attitude estimation errors

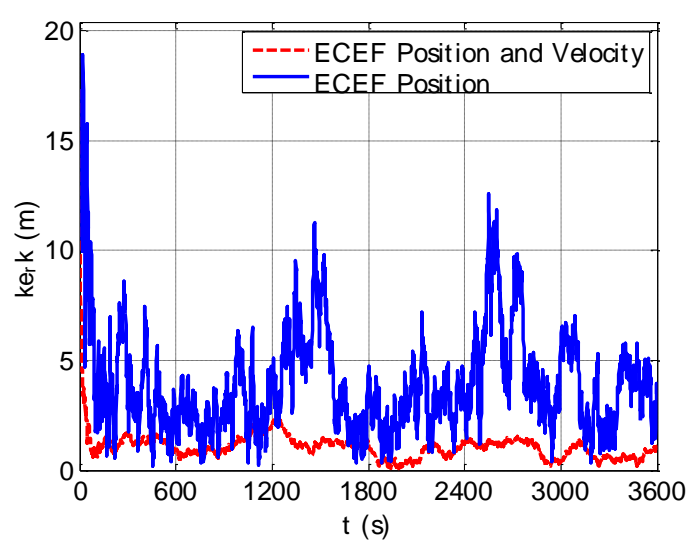

(b) Position estimation errors

Figure 3. Norms of attitude and position errors using two different measurement combinations

To prove that the loosely coupled GPS/INS integration based on the EKF using both the ECEF position and velocity can be practically implementable and its performance can be improved, the EKF is simulated for different GPS velocities using three different $\sigma_{v}$, which are $3 \times 10^{-2}, 10 \times 10^{-2}$ and $30 \times 10^{-2} \mathrm{~m} / \mathrm{s}$ for Eq. (46) under the same position error $\sigma_{r}=5 \mathrm{~m}$. The results are also obtained using Monte-Carlo simulation. Table 3 shows the averaged $J_{a}$ and $J_{r}$ values for different velocity errors using the three ECEF velocities under the same position error $\sigma_{r}=5 \mathrm{~m}$. It shows that smaller averaged $J_{a}$ and $J_{r}$ are obtained as the velocity error is smaller. It means that more accurate EKF estimation result can be achieved when more accurate velocity is used in the EKF. Even when the velocity error $\sigma_{v}=30 \times 10^{-2}$ is used with the position, $J_{r}$ in Table 3 is still smaller than, $J_{r}$ in Table 2 for the position measurement only. More accurate EKF estimation results can be obtained when both the position and velocity measurements are used together with more accurate velocity measurement. Therefore, the velocity information from the GPS receiver may improve the EKF estimation result when it is used with the ECEF position. Furthermore, as the velocity accuracy is higher, more improved EKF result can be attained.

Table 3. Averaged $J_{a}$ and $J_{r}$ values using both the position and velocity

\begin{tabular}{c|c|c}
\hline Velocity Error $\left(\sigma_{v}\right) \mathrm{m} / \mathrm{s}$ & $J_{a}$ & $J_{r}$ \\
\hline $3 \times 10^{-2}$ & $5.3430 \times 10^{3}$ & $1.0879 \times 10^{3}$ \\
\hline $10 \times 10^{-2}$ & $5.3623 \times 10^{3}$ & $1.8447 \times 10^{3}$ \\
\hline $30 \times 10^{-2}$ & $5.3789 \times 10^{3}$ & $3.0432 \times 10^{3}$ \\
\hline
\end{tabular}

Figure 4 shows the norms of the position and attitude estimation errors using different GPS velocity errors for three cases. When the standard deviation is $3 \times 10^{-2} \mathrm{~m}$, the most accurate attitude and position estimations are achieved, which the norms of the attitude and position errors go down to 0.1 degrees and $0.3 \mathrm{~m}$, respectively. As the standard deviation $\sigma_{v}$ is smaller, smaller norms of attitude and position errors are obtained. Therefore, the benefit of accurate velocity from the GPS receiver for the loosely coupled GPS/INS integration is numerically verified. 


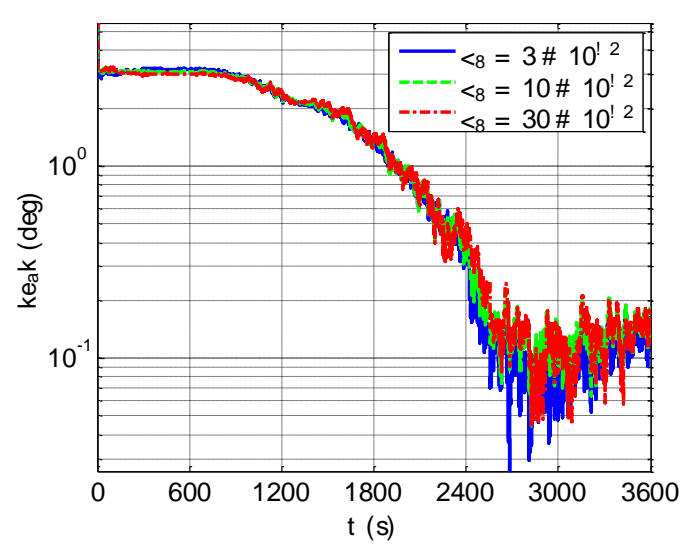

(b) Attitude estimation errors

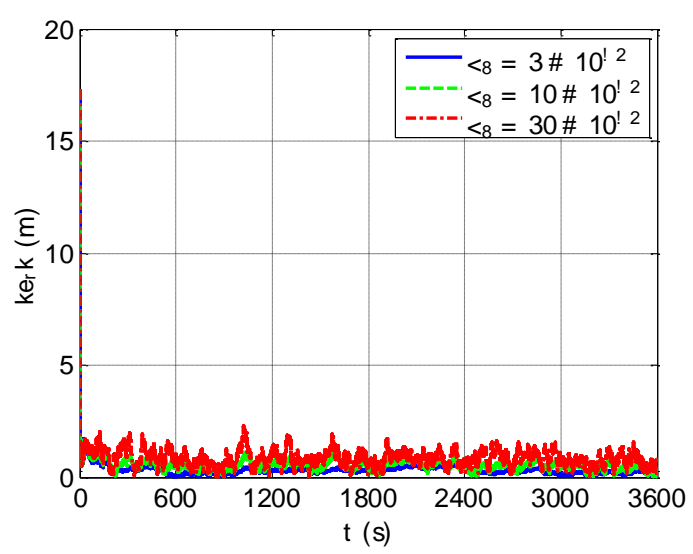

(b) Position estimation errors

Figure 4. Norms of attitude and position errors for different GPS velocity errors

\section{Conclusion}

In this paper an extended Kalman filter was derived for a loosely coupled GPS/INS integration algorithm that can take advantage of the benefits of the ECEF frame and centimeter per second-level of accuracy GPS velocity solution. The EKF is based on a quaternion parameterization of the attitude. However, straightforward implementation of the EKF using quaternion kinematics did not result in a unit quaternion estimate. The position, velocity, and bias states also follow the standard EKF additive correction while the attitude error state update is computed using a multiplicative update. The GPS navigation solutions which may consist of the position and velocity in the ECEF frame are directly used for the EKF without any coordinate transformation.

The EKF was first evaluated for different measurement types which consists of the position only, and both the position and velocity navigation solution from the GPS receiver. The EKF using both GPS the position and velocity measurements could achieve more accurate attitude and position estimation results than the EKF using the GPS position only when the velocity standard deviation error was sub-centimeter per sec level. To verify that the EKF for loosely coupled GPS/INS integration using both the GPS position and velocity measurements can be practically implementable and its estimation performance can be improved, the EKF was simulated for different GPS velocities using three different velocity standard deviations. The EKF could obtain more accurate attitude and position estimation results as the accuracy of the velocity is higher. The simulation results clearly demonstrated how a loosely coupled GPS/INS integration in an EKF formulation using the GPS position and velocity measurements can be used to estimate attitude, position, velocity and biases with improved estimation accuracy.

\section{Acknowledgement}

This work was supported by the National Research Foundation of Korea (NRF) Grant funded by the Ministry of Science and ICT (NRF-2017R1A5A1015311).

Conflicts of Interest: "The authors declare no conflict of interest." "The funders had no role in the design of the study; in the collection, analyses, or interpretation of data; in the writing of the manuscript, or in the decision to publish the results". 
Data Availability Statement: The simulation data used to support the findings of this study are available from the

corresponding author upon request

\section{Appendix A}

The partial derivative components of $F$ matrix of Eq. (40) is derived by taking the partial derivatives with respect to the state vector components of the EKF $\delta \boldsymbol{\alpha}, \mathbf{r}_{e b}^{e}, \mathbf{v}_{e b}^{e}, \boldsymbol{\beta}_{g}, \boldsymbol{\beta}_{a}, \mathbf{s}_{g}$, and $\mathbf{s}_{a}$, respectively.

$$
\begin{aligned}
& F_{11}=\frac{\partial \delta \dot{\boldsymbol{\alpha}}}{\partial \delta \boldsymbol{\alpha}}=-\left[\hat{\boldsymbol{\omega}}_{i b}^{b}\right]^{\times}=-\left[\left(I_{3 \times 3}-\hat{S}_{g}\right)\left(\tilde{\boldsymbol{\omega}}_{i b}^{b}-\hat{\boldsymbol{\beta}}_{g}\right)^{\times}\right], F_{12}=\frac{\partial \delta \dot{\boldsymbol{\alpha}}}{\partial \mathbf{r}_{e b}^{e}}=0_{3 \times 3}, F_{13}=\frac{\partial \delta \dot{\boldsymbol{\alpha}}}{\partial \mathbf{v}_{e b}^{e}}=0_{3 \times 3}, \\
& F_{14}=\left.\frac{\partial \delta \dot{\boldsymbol{\alpha}}}{\partial \Delta \boldsymbol{\beta}_{g}}\right|_{S_{g}=\hat{S}_{g}}=-\left(I_{3 \times 3}-\hat{S}_{g}\right), F_{15}=\frac{\partial \delta \dot{\boldsymbol{\alpha}}}{\partial \boldsymbol{\beta}_{a}}=0_{3 \times 3}, \quad F_{16}=\frac{\partial \delta \dot{\boldsymbol{\alpha}}}{\partial \mathbf{s}_{a}}-\left(\tilde{\Omega}_{i b}^{b}-\hat{B}_{g}\right), F_{17}=0_{3 \times 3} \\
& F_{21}=\frac{\partial \dot{\mathbf{P}}^{e}}{\partial \delta \boldsymbol{\alpha}}=0_{3 \times 3}, \quad F_{22}=\frac{\partial \dot{\mathbf{r}}_{e b}^{e}}{\partial \mathbf{r}_{e b}^{e}}=0_{3 \times 3}, F_{23}=\frac{\partial \dot{\mathbf{r}}_{e b}^{e}}{\partial \mathbf{v}_{e b}^{e}}=I_{3 \times 3}, F_{24}=\frac{\partial \dot{\mathbf{r}}_{e b}^{e}}{\partial \boldsymbol{\beta}_{g}}=0_{3 \times 3}, F_{25}=\frac{\partial \dot{\mathbf{r}}_{e b}^{e}}{\partial \boldsymbol{\beta}_{a}}=0_{3 \times 3}, \\
& F_{26}=\frac{\partial \dot{\mathbf{r}}_{e b}^{e}}{\partial \mathbf{s}_{g}}=0_{3 \times 3}, F_{27}=\frac{\partial \dot{\mathbf{r}}_{e b}^{e}}{\partial \mathbf{s}_{a}}=0_{3 \times 3}
\end{aligned}
$$

The direction cosine matrix $C_{b}^{e}(\mathbf{q})$ is written by

$$
C_{b}^{e}(\mathbf{q})=\left[C_{e}^{b}(\hat{\mathbf{q}})\right]^{T}\left[C_{e}^{b}(\delta \mathbf{q})\right]^{T}=C_{b}^{e}(\hat{\mathbf{q}})\left[I_{3 \times 3}-[\delta \boldsymbol{\alpha}]^{\times}\right]^{T}=C_{b}^{e}(\hat{\mathbf{q}})\left(I_{3 \times 3}+[\delta \boldsymbol{\alpha}]\right)
$$

Substituting the above equation yields time derivative of the velocity of Eq. (18) as

$$
\dot{\mathbf{v}}_{e b}^{e}=C_{B}^{E}(\hat{\mathbf{q}})\left(I_{3 \times 3}+[\delta \boldsymbol{\alpha}]^{\times}\right) \mathbf{f}_{i b}^{b}-2 \Omega_{i e}^{e} \mathbf{v}_{e b}^{e}-\Omega_{i e}^{e 2} \mathbf{r}_{e b}^{e}+\boldsymbol{\gamma}_{e b}^{e}
$$

The Jacobian matrix of $\boldsymbol{\gamma}_{e b}^{e}$ with respect to the position vector $\mathbf{r}_{e b}^{e}$ is given by

$$
\begin{aligned}
\nabla \gamma_{e b}^{e} & =\left[\begin{array}{lll}
\frac{\partial \gamma_{x}}{\partial x_{e}} & \frac{\partial \gamma_{x}}{\partial y_{e}} & \frac{\partial \gamma_{x}}{\partial z_{e}} \\
\frac{\partial \gamma_{y}}{\partial x_{e}} & \frac{\partial \gamma_{y}}{\partial y_{e}} & \frac{\partial \gamma_{y}}{\partial z_{e}} \\
\frac{\partial \gamma_{z}}{\partial x_{e}} & \frac{\partial \gamma_{s}}{\partial y_{e}} & \frac{\partial \gamma_{z}}{\partial z_{e}}
\end{array}\right] \\
\frac{\partial g_{x}}{\partial x_{e}}= & -\mu\left(\frac{1}{R^{3}}-\frac{3 x_{e}^{2}}{R^{5}}\right)+\frac{\mu J_{2} R_{e}^{2}}{2}\left(-\frac{3}{R^{5}}+\frac{15\left(x_{e}^{2}+z_{e}^{2}\right)}{R^{7}}-\frac{105 x_{e}^{3} z_{e}^{2}}{R^{9}}\right) \\
\frac{\partial g_{x}}{\partial y_{e}}= & \frac{3 \mu x_{e} y_{e}}{R^{5}}+\frac{\mu J_{2} R_{e}^{2}}{2}\left(\frac{15 x_{e} y_{e}}{R^{7}}-\frac{105 x_{e} y_{e} z_{e}^{2}}{R^{9}}\right) \\
\frac{\partial g_{x}}{\partial z_{e}}= & \frac{3 \mu x_{e} z_{e}}{R^{5}}+\frac{\mu J_{2} R_{e}^{2}}{2}\left(\frac{45 x_{e} z_{e}}{R^{7}}-\frac{105 x_{e} z_{e}^{3}}{R^{9}}\right) \\
\frac{\partial g_{y}}{\partial x_{e}}= & \frac{\partial g_{x}}{\partial y_{e}}, \frac{\partial g_{y}}{\partial y_{e}}=-\mu\left(\frac{1}{R^{3}}-\frac{3 y_{e}^{2}}{R^{5}}\right)+\frac{\mu J_{2} R_{e}^{2}}{2}\left(-\frac{3}{R^{5}}+\frac{15\left(y_{e}^{2}+z_{e}^{2}\right)}{R^{7}}-\frac{105 y_{e}^{3} z_{e}^{2}}{R^{9}}\right)
\end{aligned}
$$




$$
\begin{aligned}
& \frac{\partial g_{y}}{\partial z_{e}}=\frac{3 \mu y_{e} z_{e}}{R^{5}}+\frac{\mu J_{2} R_{e}^{2}}{2}\left(\frac{45 y_{e} z_{e}}{R^{7}}-\frac{105 y_{e} z_{e}^{3}}{R^{9}}\right) \\
& \frac{\partial g_{z}}{\partial x_{e}}=\frac{\partial g_{x}}{\partial z_{e}}, \frac{\partial g_{z}}{\partial y_{e}}=\frac{\partial g_{y}}{\partial z_{e}}, \frac{\partial g_{z}}{\partial z_{e}}=-\mu\left(\frac{1}{R^{3}}-\frac{3 z_{e}^{2}}{R^{5}}\right)+\frac{\mu J_{2} R_{e}^{2}}{2}\left(-\frac{9}{R^{5}}+\frac{90 z_{e}^{2}}{R^{7}}-\frac{105 z_{e}^{4}}{R^{9}}\right) \\
& F_{31}=\left.\frac{\partial \dot{\mathbf{v}}_{e b}^{e}}{\partial \delta \boldsymbol{\alpha}}\right|_{\mathbf{f}_{i b=}^{b}=\hat{\mathbf{i}}_{i b}^{b}, \mathbf{q}=\hat{\mathbf{q}}}=-C_{b}^{e}(\hat{\mathbf{q}})\left[\hat{\mathbf{f}}_{i b}^{b}\right]^{\times}, F_{32}=\left.\frac{\partial \dot{\mathbf{v}}_{e b}^{e}}{\partial \mathbf{r}_{e b}^{e}}\right|_{\mathbf{r}_{e b}^{e}=\hat{\mathbf{r}}_{e b}^{e}}=-\Omega_{i e}^{e 2}+\left.\nabla \boldsymbol{\gamma}_{e b}^{e}\right|_{\mathbf{r}_{e b}^{e}=\mathbf{r}_{e b}^{e}}, F_{33}=\frac{\partial \dot{\mathbf{v}}_{e b}^{e}}{\partial \mathbf{v}_{e b}^{e}}\left|=-2 \Omega_{i e}^{e}, F_{34}=\frac{\partial \dot{\mathbf{v}}_{e b}^{e}}{\partial \boldsymbol{\beta}_{g}}\right|=0_{3 \times 3}
\end{aligned}
$$

Substituting Eq. (23a) into Eq. (18) yields

$$
\dot{\mathbf{v}}_{e b}^{e}=C_{B}^{E}(\mathbf{q})\left(I_{3 \times 3}-S_{a}\right)\left(\tilde{\mathbf{f}}_{i b}^{b}-\boldsymbol{\beta}_{a}-\boldsymbol{\eta}_{a v}\right)-2 \Omega_{i e}^{e} \mathbf{v}_{e b}^{e}-\Omega_{i e}^{e 2} \mathbf{r}_{e b}^{e}+\boldsymbol{\gamma}_{e b}^{e}
$$

Using the above equation the partial derivative components of the third row of $F$ matrix are obtained by

$$
\begin{aligned}
& F_{35}=\left.\frac{\partial \dot{\mathbf{v}}_{e b}^{e}}{\partial \boldsymbol{\beta}_{a}}\right|_{\mathbf{q}=\hat{\mathbf{q}}, \mathbf{s}_{a}=\hat{\mathbf{s}}_{a}}=-C_{b}^{e}(\hat{\mathbf{q}})\left(I_{3 \times 3}-\hat{S}_{a}\right), F_{36}=\frac{\partial \dot{\mathbf{v}}_{e b}^{e}}{\partial \mathbf{s}_{g}}=0_{3 \times 3}, F_{37}=\frac{\partial \dot{\mathbf{v}}_{e b}^{e}}{\partial \mathbf{s}_{a}} \mid=-C_{b}^{e}(\hat{\mathbf{q}})\left(\tilde{F}_{i b}^{b}-\hat{S}_{a}\right) \\
& F_{41}=\frac{\partial \dot{\boldsymbol{\beta}}_{g}}{\partial \delta \boldsymbol{\alpha}}=0_{3 \times 3}, F_{42}=\frac{\partial \dot{\boldsymbol{\beta}}_{g}}{\partial \mathbf{r}_{e b}^{e}}=0_{3 \times 3}, F_{43}=\frac{\partial \dot{\boldsymbol{\beta}}_{g}}{\partial \mathbf{v}_{e b}^{e}}=0_{3 \times 3}, F_{44}=\frac{\partial \dot{\boldsymbol{\beta}}_{g}}{\partial \boldsymbol{\beta}_{g}}=0_{3 \times 3}, F_{45}=\frac{\partial \dot{\boldsymbol{\beta}}_{g}}{\partial \boldsymbol{\beta}_{a}}=0_{3 \times 3}, \\
& F_{46}=\frac{\partial \dot{\boldsymbol{\beta}}_{g}}{\partial \mathbf{s}_{g}}=0_{3 \times 3}, F_{47}=\frac{\partial \dot{\boldsymbol{\beta}}_{g}}{\partial \mathbf{s}_{a}}=0_{3 \times 3} \\
& F_{51}=\frac{\partial \dot{\boldsymbol{\beta}}_{g}}{\partial \delta \boldsymbol{\alpha}}=0_{3 \times 3}, F_{52}=\frac{\partial \dot{\boldsymbol{\beta}}_{g}}{\partial \mathbf{r}_{e b}^{e}}=0_{3 \times 3}, F_{53}=\frac{\partial \dot{\boldsymbol{\beta}}_{g}}{\partial \mathbf{v}_{e b}^{e}}=0_{3 \times 3}, F_{54}=\frac{\partial \dot{\boldsymbol{\beta}}_{g}}{\partial \boldsymbol{\beta}_{g}}=0_{3 \times 3}, F_{55}=\frac{\partial \dot{\boldsymbol{\beta}}_{g}}{\partial \boldsymbol{\beta}_{a}}=0_{3 \times 3}, \\
& F_{56}=\frac{\partial \dot{\boldsymbol{\beta}}_{g}}{\partial \mathbf{s}_{g}}=0_{3 \times 3}, F_{57}=\frac{\partial \dot{\boldsymbol{\beta}}_{g}}{\partial \mathbf{s}_{a}}=0_{3 \times 3} \\
& F_{61}=\frac{\partial \dot{\mathbf{s}}_{g}}{\partial \delta \boldsymbol{\alpha}}=0_{3 \times 3}, F_{62}=\frac{\partial \dot{\mathbf{s}}_{g}}{\partial \mathbf{r}_{e b}^{e}}=0_{3 \times 3}, F_{63}=\frac{\partial \dot{\mathbf{s}}_{g}}{\partial \mathbf{v}_{e b}^{e}}=0_{3 \times 3}, F_{64}=\frac{\partial \dot{\mathbf{s}}_{g}}{\partial \boldsymbol{\beta}_{g}}=0_{3 \times 3}, F_{65}=\frac{\partial \dot{\mathbf{s}}_{g}}{\partial \boldsymbol{\beta}_{a}}=0_{3 \times 3}, \\
& F_{76}=\frac{\partial \dot{\mathbf{s}}_{g}}{\partial \mathbf{s}_{g}}=0_{3 \times 3}, F_{77}=\frac{\partial \dot{\mathbf{s}}_{a}}{\partial \mathbf{s}_{a}}=0_{3 \times 3}, F_{67}=\frac{\partial \dot{\mathbf{s}}_{g}}{\partial \mathbf{s}_{a}}=0_{3 \times 3} \\
& F_{71}=\frac{\partial \dot{\mathbf{s}}_{a}}{\partial \delta \boldsymbol{\alpha}}=0_{3 \times 3}, F_{72}=\frac{\partial \dot{\mathbf{s}}_{a}}{\partial \mathbf{r}_{e b}^{e}}=0_{3 \times 3}, F_{73}=\frac{\partial \dot{\mathbf{s}}_{a}}{\partial \mathbf{v}_{e b}^{e}}=0_{3 \times 3}, F_{74}=\frac{\partial \dot{\mathbf{s}}_{a}}{\partial \boldsymbol{\beta}_{g}}=0_{3 \times 3}, F_{75}=\frac{\partial \dot{\mathbf{s}}_{a}}{\partial \boldsymbol{\beta}_{a}}=0_{3 \times 3}, \\
& F_{3},
\end{aligned}
$$

\section{References}

[1] Parkinson , B. W.; Spilker Jr., J. J., et al., Global Positioning System: Theory and Applications, vols. I \& II, AIAA, Inc., New York, 1996.

[2] Kaplan, E. D., Understanding GPS, Principle and Applications, Artech House, Boston, 1996.

[3] Crassidis, J. L., "Sigma-Point Kalman Filtering for Integrated GPS and Inertial Navigation,” AIAA Guidance, Navigation, and Control Conference and Exhibit 15-18, August 2005, San Francisco, California.

[4] Crassidis, J. L., "Sigma-Point Kalman Filtering for Integrated GPS and Inertial Navigation," IEEE Transactions on Aerospace and Electronic Systems, 2006, Vol. 42, Issue 2, pp. 750-756, T-AES/42/2/876457. 
[5] Van Graas, F., and Soloviev A., A., "Precise Velocity Estimation Using a Stand-Alone GPS Receiver," The Journal of Navigation, Volume 64 , Issue 2 , April 2011 , pp. 311-325.

[6] P., Freda, Angrisano, A., Gaglione, S. and Troisi, S., "Time-Differenced Carrier Phases Technique for Precise GPS Velocity Estimation,” GPS Solutions, 2015, Vol. 19, Issue 2, pp. 335-341.

[7] H., Le Nhat and N., Vinh Hao, "Loosely Coupled GPS/INS Integration with Kalman filtering for Land Vehicle Applications," 2012 International Conference on Control, Automation and Information Sciences (ICCAIS), 26-29 Nov. 2012, Ho Chi Minh City, Vietnam, pp. 90-95.

[8] Groves, P. D., Principles of GPS, Inertial, and Multisensor Integrated Navigation Systems, $2^{\text {nd }}$ Ed, Artech House, Ch. 14, 2013.

[9] Falco, G., Einicke, G. A., Malos, J. M. and Dovis F., Performance analysis of constrained loosely Coupled GPS/INS integration solutions, Sensors, 2012, Vol. 12, No. 11, pp. 15983-16007.

[10] Wei, M. and SCHWARZ, K. P., “A Strapdown Inertial Algorithm Using an Earth-Fixed Cartesian Frame," Navigation: Journal of The Institute of Navigation, 1990, Vol. 37, No. 2, pp. 153-167, 10.1002/j.2161-4296.1990.tb01544.x.

[11] Ezzaldeen, E., Zhou, J., Zhang J. and Loffeld O., A new loosely coupled DCM based GPS/INS Integration Method," Navigation: Journal of The Institute of Navigation, 2012, Vol. 59, Issue 2, pp. 93-106, doi.org/10.1002/navi.7.

[12] Gross, J. N., Gu, Y., Gururajan, S., and Napolitano, M. R., "Flight-Test Evaluation of Sensor Fusion Algorithms for Attitude Estimation,” IEEE Transactions on Aerospace and Electronic Systems, 2012, Vol. 48, No. 3, pp. 2128-2139, 10.1109/TAES.2012.6237583.

[13] Crassidis, J. L. and Junkins, J. L., Optimal Estimation of Dynamic Systems, CRC press LLC, $1^{\text {st }}$ Ed., Ch.7, 2004.

[14] Freda, P., Angrisano A., Gaglione S., Troisi S., "Time-differenced carrier phases technique for precise GPS velocity estimation," GPS Solut, 2015, Vol. 9, pp. 335-341, doi:10.1007/s10291-014-0425-1.

[15] E. J. Lefferts, F. L. Markley, and M. D. Shuster, "Kalman Filtering for Spacecraft Attitude Estimation," Journal of Guidance, Control, and Dynamics, 1982, Vol. 5, No. 5, pp. 417-429, 10.2514/3.56190.

[16] Hamilton, W. R., Elements of Quaternions, Longmans, Green and Co., London, England, 1866.

[17] Titterton, D. H. and Weston, J. L., Strapdown Inertial Navigation Technology, $2^{\text {nd }}$ Ed., The Institution of Electrical Engineers, 2004.

[18] Loan, C. V., "Computing Integrals Involving the Matrix Exponential," IEEE Transactions on Automatic Control, 1978, Vol. AC-23, No. 3, pp. 396-404, 10.1109/TAC.1978.1101743. 